\title{
Preservation Properties for the Discrete Mean Residual Life Ordering
}

\author{
Abdulhakim Al-Babtain \\ Department of Statistics and Operations Research, King Saud University \\ hakim@ksu.edu.sa \\ Abdul Hadi Nabih Ahmed \\ Department of Mathematical Statistics, Cairo University, Egypt \\ drhadi2010@gmail.com
}

\begin{abstract}
The purpose of this paper is to prove several preservation properties of stochastic comparisons based on the discrete mean residual life ordering d-MRL under the reliability operations of convolutions, mixtures. Finally we introduce a discrete renewal process application.
\end{abstract}

Keywords: Discrete Mean Residual Life, Convolution, Mixtures, Weak convergence.

\section{Introduction and Motivation}

Discrete lifetimes usually arise through grouping or finite-precision measurement of continuous time phenomena. They may also be found natural choice where failure may occur only due to incoming shocks. Parametric models for discrete life distributions may be found in Bain (1991), Adams and Watson (1989) and Xekalaki (1983). Nonparametric families of discrete life distributions has been considered in the reliability literature mainly in connection with shock models leading to various continuous-time ageing families see Barlow and Proschan, (1975), Among others, have studied interrelations and closure properties of some nonparametric ageing families of distributions having a finite support. Related partial orders have been considered by Abouammoh (1990).

Recently, Nanda and Sengupta (2005) have discussed reversed hazard rate in discrete setup and obtained several interesting results.

Let $X$ be a non-negative random variable with probability mass function (p.m.f) given by:

$$
f(x)=P(X=x), \quad x \in N=\{0,1, \ldots\}
$$

the cumulative distribution function

and the survival function

$$
F(x)=P(X \leq x)=\sum_{i=0}^{x} f(i) \text { for all } x \in N
$$

$$
\bar{F}(x)=1-F(x)=\sum_{i=x+1}^{\infty} f(i) \text { for all } x \in N
$$

In particular, if $f(0)=\operatorname{Pr}\{X=0\}=0$, or a counting random variable $\mathrm{X}$ has a support on $N_{+}=\{1,2, \ldots\}$, we say that the discrete distribution is zero- truncated. Recently, Pavlova et al (2006) defined discrete hazard rate and discrete mean residual lifetime of $F$ by

$$
h_{F}(x)=\frac{f(x)}{f(x)+F(x)} \text { for all } x \in N \text {, and } P\{X \geq x\}>0 \text {, }
$$


and

$$
M_{F}(x)=E(X-x \mid X>x)=\left(\frac{\sum_{i=x}^{\infty} \bar{F}(i)}{F(x)}\right) \text { for all } x \in N, \text { and } \bar{F}(x)>0
$$

There is an abundance of literature on continuous life distributions used in modeling failure data. However, very little has appeared in the literature for discrete failure models. Discrete failure data arise in various common situations. Consider the following examples:

a) A device can be monitored only once per time period and the observation are taken as the number of time periods successfully completed prior to the failure of the device.

b) A piece of equipment operates in cycles. In this case the random variable of interest is the successful number of cycles before the failure. For instance, the number of flashes in a car flasher prior to failure of the device.

c) In some situations the experimenter groups or finite precision measurement of continuous time phenomena.

Shaked et al. (1994, 1995) state that discrete failure rates arise in several common situations in reliability theory where clock time is not the best scale on which to describe lifetime. For example, in weapons reliability, the number of rounds fixed until failure is more important than age in failure. They also showed the usefulness of these functions for modeling imperfect repair and for characterizing ageing in the discrete setting. For more applications of discrete models in reliability and survival analysis, see Padgett and Spurrier (1985) and Ebrahimi (1986). More precise concepts of discrete reliability theory have been settled by Salvia and Bollinger (1982), Roy and Gupta (1992) examined classification of discrete life distributions and they introduced the concepts of second rate of failure to maintain analogy with the continuous aging classes. Salvia (1996) presents some results on discrete mean residual life.

Similar to continuous distributions, discrete distributions can also be classified by the properties of the failure rates, the mean residual lifetimes, and survival functions of discrete distributions. Some commonly used classes of discrete distributions include the classes of discrete decreasing failure rate (d-DF R), discrete decreasing failure rate average (d-DFRA), discrete new worse than used (d-NWU), discrete increasing mean residual lifetime (d-IMRL), discrete harmonic new worse than used in expectation (dHNWUE), and their dual ones including the classes of discrete increasing failure rate (dIFR), discrete increasing failure rate average (d-IFRA), discrete new better than used (d$\mathrm{NBU}$ ), discrete decreasing mean residual lifetime (d-DMRL), and discrete harmonic new better than used in expectation (d-HNBUE):

These classes of discrete distributions have been used extensively in different fields of statistics and probability such as insurance, finance, reliability, survival analysis, and others. See, for example, Barlow and Proschan (1975), Cai and Kalashnikov (2000), Cai and Willmot (2005), Fagiuoli and Pellerey (1994), Hu et al. (2003), Johnson et al. (1993), Kijima (2003), Shaked and Shanthikumar (1994), Shaked et al. (1995), Willmot and Cai (2001), Willmot and Lin (2000), Willmot et al. (2005), and references therein. 


\section{Preliminaries}

In this section we present definitions, notation, and basic facts used throughout the paper. We use "increasing" in place of "non-decreasing" and "decreasing" in place of "nonincreasing". Let $X$ and $Y$ be two non-negative random variables with $\mathrm{F}$ and $\mathrm{G}$ as their respective distribution functions. Let $\bar{F}(t)=1-F(t)$, and $\bar{G}(t)=1-G(t)$. We will assume that $\bar{F}(0)=\bar{G}(0)=1$ in all cases.

\section{Definition 2.1}

The random variable $X$ is said to have a smaller discrete mean residual lifetime than that of $Y$, written $X \leq_{(d-M R L)} Y$, if

$$
\left(\frac{\sum_{i=x}^{\infty} \bar{F}(i)}{\bar{F}(x)}\right) \leq\left(\frac{\sum_{i=x}^{\infty} \bar{G}(i)}{\bar{G}(x)}\right) \text {, for all } x \in N
$$

Note that, (2.1) is equivalent to saying

$$
\left(\frac{\sum_{i=x}^{\infty} \bar{F}(i)}{\sum_{i=x}^{\infty} \bar{G}(i)}\right) \text { is decreasing in } x \text { for all } x \in N \text {. }
$$

\section{Definition 2.2}

The random variable $X$ has a smaller discrete hazard rate than that of $Y$, written $X \leq_{d-H} Y$, if

$$
\frac{\bar{F}(i)}{\bar{G}(i)} \text { is increasing in } x \text { for all } x \in N \text {. }
$$

\section{Definition 2.3}

The distribution $F$ is called discrete decreasing failure rate (discrete increasing failure rate) or $d-D F R(d-I F R)$ if its failure rate $h_{F}(x)=\frac{f(x)}{f(x)+F(x)}$ is non-increasing (nondecreasing) for $x \in N_{+}$and $f(X)+F(x)>0$.

We notice that the discrete decreasing failure rate life distributions govern,

a) In the grouped data case, the number of periods until failure of a device governed by a DFR life distribution.

b) The number of seasons a TV show is run before being cancelled. Thus the d-DFD life distributions are of great significance in spite of their relative neglect in the reliability literature.

\section{Definition 2.4}

The distribution $\mathrm{F}$ is called discrete new better than used in expectation or $d-N B U E$ (discrete new worse than used in expectation or $d-N W U E$ ) if

$$
\sum_{i=x}^{\infty} \bar{F}(i) \leq(\geq) \bar{F}(x) \sum_{j=x}^{\infty} \bar{F}(j), \quad \text { for all } x \in N
$$




\section{Definition 2.5}

The distribution $\mathrm{F}$ is called discrete decreasing mean residual lifetime or $d-D M R L$ if its mean residual lifetime $M_{F}(x)=\left(\frac{\sum_{i=x}^{\infty} \bar{F}(i)}{\bar{F}(x)}\right)$; for $x \in N$ is decreasing in $x \in N$. The following two definitions will be used in sequel.

\section{Definition 2.6}

A probability vector $\underline{\alpha}=\left(\alpha_{1}, \alpha_{2}, \ldots, \alpha_{n}\right)$ is said to be smaller than the probability vector $\beta=\left(\beta_{1}, \beta_{2}, \ldots, \bar{\beta}_{n}\right)$, in the sense of the discrete likelihood ratio order, denoted by

$$
\alpha \leq_{d-L R} \beta \quad \text { if } \quad \frac{\beta_{i}}{\alpha_{i}} \leq \frac{\beta_{j}}{\alpha_{j}} \quad \text { for all } 1 \leq i \leq j \leq n .
$$

\section{Definition 2.7}

A function $g: R^{2} \rightarrow[0, \infty)$ is said to be log-concave if

$$
g\left(x_{1}, y_{1}\right) g\left(x_{2}, y_{2}\right)-g\left(x_{1}, y_{2}\right) g\left(x_{2}, y_{1}\right) \geq 0 \text { whenever } x_{1}<x_{2}, y_{1}<y_{2} \text {. }
$$

The reminder of this paper is organized as follows. In section 3 we present the main results concerning the statistical properties of the discrete mean residual life ordering, such as convolution, mixtures and convergence in distributions. Section 4 contains two results concerning discrete renewal process in connection with the orders in the paper.

\section{Main Results}

In this section we present preservation results for the discrete mean residual life ordering. We point out that similar results hold for both the hazard rate ordering and the likelihood ratio ordering. We begin by showing that the discrete mean residual life ordering is preserved under weak limits in distributions.

\section{Theorem 3.1}

The discrete mean residual life ordering $\left(\leq_{d-M R L}\right)$ preserves the weak convergence property.

\section{Proof}

Suppose $\left\{F_{n}\right\}$ and $\left\{G_{n}\right\}$ gconverge weakly to $F$ and $G$ and that $F_{n} \leq_{d-M R L} G_{n}$. Then if $y$ is a continuity point of both $F$ and $G$, it follows that $\mu_{F}(y) \leq \mu_{G}(y)$. Thus, $\mu_{F}(y)>\mu_{G}(y)$ is possible only if $y$ is a discontinuity point of either $F$ or $G$. Such discontinuity points are at most countable, so there exist continuity points $x_{n}$ of $F$ and $G$ for which $x_{n} \downarrow y$ as $n \rightarrow$ $\infty$. Consequently, appealing to the right-continuity property of distribution functions

$$
\mu_{F}(y)=\lim _{n \rightarrow \infty} \mu_{F}\left(x_{n}\right) \leq \lim _{n \rightarrow \infty} \mu_{G}\left(x_{n}\right)=\mu_{G}(y),
$$

whence a contradiction. The following result shows that the mean residual life ordering is preserved under convolutions.

For next results we shall use the notation $a_{i}$ and $b_{i}$ to replace $\bar{F}(i)$ and $\bar{G}(i)$ respectively. 


\section{Theorem 3.2}

Let $X_{1}, X_{2}$ and $Y$ be three nonnegative discrete random variables, where $Y$ is independent of both $X_{1}, X_{2}$, also let $Y$ have a probability mass function $g$. Then $X_{1} \leq_{d-M R L} X_{2}$ and $g$ is log-concave imply that $X_{1}+Y \leq_{d-M R L} X_{2}+Y$

\section{Proof}

We have to show that

$\frac{\sum_{u=0}^{\infty} \sum_{x=0}^{\infty} g(t-u) a_{x+u}}{\sum_{u=0}^{\infty} \sum_{x=0}^{\infty} g(t-u) b_{x+u}} \geq \frac{\sum_{u=0}^{\infty} \sum_{x=0}^{\infty} g(s-u) a_{x+u}}{\sum_{u=0}^{\infty} \sum_{x=0}^{\infty} g(s-u) b_{x+u}}$, for all $0 \leq s \leq t$.

Or equivalently,

$$
\left|\begin{array}{ll}
\sum_{u=0}^{\infty} \sum_{x=0}^{\infty} g(t-u) b_{x+u} & \sum_{u=0}^{\infty} \sum_{x=0}^{\infty} g(s-u) a_{x+u} \\
\sum_{u=0}^{\infty} \sum_{x=0}^{\infty} g(t-u) b_{x+u} & \sum_{u=0}^{\infty} \sum_{x=0}^{\infty} g(t-u) a_{x+u}
\end{array}\right| \geq 0 .
$$

Next, by the well known basic composition formula (Karlin, 1968, p.17, the left side of (3.2) is equal to

$$
\sum \sum_{u_{1}<u_{2}}\left|\begin{array}{ll}
g\left(s-u_{1}\right) & g\left(s-u_{2}\right) \\
g\left(t-u_{1}\right) & g\left(t-u_{2}\right)
\end{array}\right|\left|\begin{array}{|ll}
\sum_{x=0}^{\infty} b_{x+u_{1}} & \sum_{x=0}^{\infty} a_{x+u_{1}} \\
\sum_{x=0}^{\infty} b_{x+u_{2}} & \sum_{x=0}^{\infty} a_{x+u_{2}}
\end{array}\right|
$$

the conclusion now follows if we note that the first determinant is non-negative since $\mathrm{g}$ is log-concave, and that the second determinant is nonnegative since $X_{1} \leq_{d-M R L} X_{2}$.

\section{Corollary 3.3}

If $X_{1} \leq_{d-M R L} Y_{1}$ and $X_{2} \leq_{d-M R L} Y_{2}$ where $X_{1}$ is independent of $X_{2}$ and $Y_{1}$ independent of $Y_{2}$ then the following statements hold:

a) If $X_{1}$ and $Y_{2}$ have log-concave probability mass functions, then $X_{1}+X_{2} \leq_{d-M R L} Y_{1}+$ $Y_{2}$.

b) If $X_{2}$ and $Y_{1}$ have log-concave probability mass functions, then $X_{1}+X_{2} \leq_{d-M R L} Y_{1}+$ $Y_{2}$.

\section{Proof}

The following chain of inequalities establish (a):

$$
X_{1}+X_{2} \leq_{\{d-M R L\}} X_{1}+Y_{2} \leq_{\{d-M R L\}} Y_{1}+Y_{2}
$$

the proof of (a) is similar.

Let $X(\theta)$ be a non- negative discrete random variable having distribution function $F_{\theta}$ and let $\theta_{i}$ be a random variable having distribution $G_{i}(i=1 ; 2)$ and support $R^{+}$. The following theorem shows that the $d-M R L$ ordering is preserved under mixtures. 


\section{Theorem 3.4}

Let $\left\{X(\theta), \theta \in R^{+}\right\}$be a family of random variables independent of $\Theta_{1}$ and $\Theta_{2}$. If $\Theta_{1} \leq_{L R} \Theta_{2}$ and if $X\left(\Theta_{1}\right) \leq_{d-M R L} X\left(\Theta_{2}\right)$ whenever $\theta_{1} \leq \theta_{2}$, then $X\left(\theta_{1}\right) \leq_{d-M R L} X\left(\theta_{2}\right)$.

\section{Proof}

Let $F_{i}$ be the distribution function of $X\left(\Theta_{i}\right)$ with $i=1,2$. We know that

$$
\bar{F}_{i}(x)=\int_{0}^{\infty} \bar{F}_{\theta}(x) d G_{i}(\theta)
$$

In the light of 2.2 we shall prove that

$$
\Phi(i, k)=\sum_{u=0}^{k} F_{i}(k-u)
$$

is $T P_{2}$ in $(i, k)$. We notice that

$$
\begin{array}{r}
\Phi(i, k)=\sum_{u=0}^{k} F_{i}(k-u)=\sum_{u=0}^{k} F_{i}(k-u) \int_{0}^{\infty} \bar{F}_{\theta}(k-u) d G_{i}(\theta) \\
=\int_{0}^{\infty} g_{i}(\theta) \sum_{u=0}^{k} F_{\theta}(k-u) d \theta=\int_{0}^{\infty} g_{i}(\theta) \Psi(\theta, k) d \theta
\end{array}
$$

By assumption $X\left(\theta_{1}\right) \leq_{d-M R L} X\left(\theta_{2}\right)$ whenever we have $\theta_{1} \leq \theta_{2}$, we have $\Psi(\theta, k)$ is $T P_{2}$ in $(\theta, k)$, while the assumption $\Theta_{1} \leq_{L R} \Theta_{2}$ implies that $g_{i}(\theta)$ is $T P_{2}$ in $(i, \theta)$. Thus the assertion follows from the basic composition formula (see, Karlin, 1968).

Let $\underline{\alpha}=\left(\alpha_{1}, \alpha_{2}, \ldots, \alpha_{n}\right)$ be less ordered than $\beta=\left(\beta_{1}, \beta_{2}, \ldots, \beta_{n}\right)$, in the sense of the discrete likelihood ratio ordering. We shall compare the distribution function of

$$
F(x)=\alpha_{1} F_{1}(x)+\cdots+\alpha_{n} F_{n}(x)
$$

for a random variable $X$, and

$$
G(x)=\beta_{1} F_{1}(x)+\cdots+\beta_{n} F_{n}(x)
$$

for a random variable $Y$.

\section{Theorem 3.5}

Let $X_{1}, \ldots, X_{n}$ be a collection of discrete random variables with corresponding distribution functions $F_{1}, \ldots, F_{n}$ such that

$$
X_{1} \leq_{d-M R L} X_{2} \leq_{d-M R L} \cdots \leq_{d-M R L} X_{n}
$$

and let $\underline{\alpha}=\left(\alpha_{1}, \alpha_{2}, \ldots, \alpha_{n}\right)$ and $\underline{\beta}=\left(\beta_{1}, \beta_{2}, \ldots, \beta_{n}\right)$ be two probability vectors with

$\alpha \leq_{d-M R L} \beta$

Then $\mathrm{X} \leq \_\{\mathrm{d}-\mathrm{MRL}\} \mathrm{Y}$.

\section{Proof}

We need to establish

$$
\frac{\sum_{x=0}^{\infty} \sum_{i=1}^{n} \beta_{i} a_{t+x}^{i}}{\sum_{x=0}^{\infty} \sum_{i=1}^{n} \alpha_{i} a_{t+x}^{i}} \leq \frac{\sum_{t=0}^{\infty} \sum_{i=1}^{n} \beta_{i} a_{t+y}^{i}}{\sum_{t=0}^{\infty} \sum_{i=1}^{n} \alpha_{i} a_{t+y}^{i}}, \text { for all } 0 \leq x \leq y .
$$


Multiplying by the denominators and canceling out equal terms shows that (3.4) is equivalent to

$$
\sum_{i=0}^{n} \sum_{j=1}^{n} \beta_{i} \alpha_{j} \sum_{u=0}^{\infty} a_{u+x}^{i} \sum_{v=0}^{\infty} a_{v+y}^{j} \leq \sum_{i=0}^{n} \sum_{j=1}^{n} \beta_{i} \alpha_{j} \sum_{u=0}^{\infty} a_{u+y}^{i} \sum_{v=0}^{\infty} a_{v+x}^{j},
$$

or when $j>i$ we get

$$
\begin{gathered}
\sum_{i=0}^{n} \sum_{j=1}^{n}\left[\beta_{i} \alpha_{j} \sum_{u=0}^{\infty} a_{u+x}^{i} \sum_{v=0}^{\infty} a_{v+y}^{j}+\beta_{j} \alpha_{i} \sum_{u=0}^{\infty} a_{u+x}^{j} \sum_{v=0}^{\infty} a_{v+y}^{i}\right] \\
\leq \sum_{i=0}^{n} \sum_{j=1}^{n}\left[\beta_{i} \alpha_{j} \sum_{u=0}^{\infty} a_{u+y}^{i} \sum_{v=0}^{\infty} a_{v+x}^{j}\right. \\
\left.+\beta_{j} \alpha_{i} \sum_{u=0}^{\infty} a_{u+y}^{j} \sum_{v=0}^{\infty} a_{v+x}^{i}\right]
\end{gathered}
$$

Now, for each fixed pair $(i, j)$ with $i<j$ we have

$$
\begin{aligned}
\beta_{i} \alpha_{j} \sum_{v=0}^{\infty} a_{v+y}^{i} \sum_{u=0}^{\infty} a_{u+x}^{j}+\beta_{j} \alpha_{i} \sum_{v=0}^{\infty} a_{v+y}^{i} \sum_{u=0}^{\infty} a_{u+x}^{i}-\beta_{i} \alpha_{j} \sum_{u=0}^{\infty} a_{u+x}^{i} \sum_{v=0}^{\infty} a_{v+y}^{i} \\
-\beta_{i} \alpha_{j} \sum_{u=0}^{\infty} a_{u+x}^{j} \sum_{v=0}^{\infty} a_{v+y}^{i} \\
=\left(\beta_{i} \alpha_{j}-\beta_{j} \alpha_{i}\right)\left[\sum_{v=0}^{\infty} a_{v+y}^{i} \sum_{u=0}^{\infty} a_{u+x}^{j}\right. \\
\left.-\sum_{u=0}^{\infty} a_{u+x}^{i} \sum_{v=0}^{\infty} a_{v+y}^{j}\right]
\end{aligned}
$$

which is nonnegative because both terms are nonnegative by assumption. This completes the proof.

In any attempt to construct new discrete mean residual life ordered random variables from known ones, the following theorem might be used.

\section{Theorem 3.6}

Let $X_{1}, X_{2}, \ldots$ and $X_{1}, X_{2}, \ldots$ are sequences of independent random variables with $X_{i} \leq_{d-M R L} Y_{i}$ and $X_{i}, Y_{i}$ have log- concave probability mass functions for all $i$, then

$$
\sum_{i=1}^{n} X_{i} \leq_{d-M R L} \sum_{i=1}^{n} Y_{i}, n=1,2, \ldots
$$

\section{Proof}

We shall prove the theorem by induction. Clearly, the result is true for $n=1$. Assume that the result is true for $p=n-1$, this means that

$$
\sum_{i=1}^{n-1} X_{i} \leq_{d-M R L} \sum_{i=1}^{n-1} Y_{i}
$$


Note that each of the two sides of (3.8) has log-concave probability mass function (see, e.g., Karlin, 1968, p.128). Appealing to Corollary 3.3, the result follows.

\section{Remark 3.7}

Similar results hold if the discrete mean residual life ordering is replaced by the discrete hazard rate ordering in Theorem 3.2 and its corollary, Theorem 3.4 and Theorem 3.5. To demonstrate the usefulness of the above results in recognizing discrete mean residual life ordered random variables, we consider the following.

\section{Example 3.8}

Let $X_{\underline{p}}$ denote the convolution of $\mathrm{n}$ geometric distributions with parameters $p_{1}, p_{2}, \ldots, p_{n}$ respectively. Assume without loss of generality that $p_{1} \geq p_{2} \geq \cdots \geq p_{n}$. Since geometric probability mass functions are log-concave, Theorem 3.5 implies that $X_{\underline{p}} \leq_{d-M R L} X_{q}$ whenever $P_{i} \geq q_{j}, i=1,2, \ldots, n$.

\section{Example 3.9}

Let $X_{p}$ be as described in Example (3.7). An application theorem (3.4) immediately yields

$$
\sum_{i=1}^{\infty} \alpha_{i} X_{p_{i}} \leq_{d-M R L} \sum_{i=1}^{\infty} \beta_{i} X_{p_{i}}
$$

for every two probability vectors $\underline{\alpha}$ and $\underline{\beta}$ such that $\underline{\alpha} \leq_{d-M R L} \underline{\beta}$. Another application of Theorem 3.4 is contained in:

\section{Example 3.10}

Let $X_{\underline{p}}$ and $X_{\underline{q}}$ as be given in Example 3.7 for $0 \leq \theta_{1} \leq \theta_{2} \leq 1$ and $\theta_{1}+\theta_{2}=1$, we have

$$
\theta_{1} X_{\underline{p}}+\theta_{2} X_{\underline{q}} \leq_{d-M R L} \theta_{2} X_{\underline{p}}+\theta_{1} X_{\underline{q}}
$$

It is remarkable that the above example can be generalized to higher dimensions, with obvious modifications in $\underline{\alpha}$ and $\beta$.

Let $F_{t}(x)=\left(\frac{F(x+t)}{F(t)}\right)$ be the conditional reliability of a unit of age $t$, then we have the following characterization of $d-D M R L$ distributions.

\section{Theorem 3.11}

$F \geq_{d-M R l} F_{t}$ for all $t \geq 0$ if and only if $F$ is $d-D M R l$.

\section{Proof}

Observe that $F \geq_{d-M R l} F_{t}$ if and only if

$$
\frac{\sum_{k=x}^{\infty} \bar{F}_{t}(k)}{\bar{F}_{t}(x)} \leq \frac{\sum_{k=x}^{\infty} \bar{F}(k)}{\bar{F}(x)},
$$


but the latter is equivalent to $\epsilon(t+x) \leq \epsilon(x)$ that is $\epsilon(t)$ is decreasing. This completes the proof.

\section{A discrete renewal process application}

Let $\left(N_{F}(t), t \geq 0\right)$ and $\left(N_{G}(t), t \geq 0\right)$ denotes two renewal processes having inter-arrival distributions $F$ and $G$, respectively.

\section{Theorem 4.1}

$$
\text { If } F \geq_{d-M R l} G \text {, then } N_{F}(t) \geq_{d-V} N_{G}(t) .
$$

\section{Proof}

The Theorem follows by mincing the elegant proofs of Lemma 8.5.5 and theorem 8.6.4 of Ross (1984) and the fact that

$$
E\left(\sum_{i=1}^{N_{F}(t)+1} X_{i}\right)=E\left(X_{1} \mid X_{1}>t\right) \geq E\left(Y_{1} \mid Y_{1}>t\right)=E\left(\sum_{i=1}^{N_{G}(t)+1} X_{i}\right)
$$

where $\left(X_{i}\right)$ and $\left(Y_{i}\right)$ are two sequences of independent identically distributed random variables having $\mathrm{F}$ and $\mathrm{G}$ as their respective distributions.

A version of the arguments used to prove corollary 3.16 and Theorem 3.17 in chapter (6) of Barlow and Proschan (1975) can be used to show that the following are valid.

\section{Corollary 4.2}

Let $F \geq_{d-M R l} G$, and $0 \leq h(1) \leq h(2) \leq \cdots$, then

$$
\sum_{n=1}^{\infty} h(n) F_{(n)}(t) \leq \sum_{n=1}^{\infty} h(n) G_{(n)}(t)
$$

\section{Theorem 4.3}

If $F \geq_{d-M R l} G, c(k)$ is convex increasing and $c(0)=0$, then

$$
\sum_{k=0}^{\infty} c(k) P\left(N_{F}(t)=k\right) \leq \sum_{k=0}^{\infty} c(k) P\left(N_{G}(t)=k\right),
$$

for an application of Theorem 4.2 in minimizing the expected shortage in spare part one may consult Barlow and Proschan (1975).

\section{Acknowledgment}

This project was supported by King Saud University, Deanship of Scientific Research, and Collage of Sciences Research Center.

\section{References}

1. Abouammoh A. M. (1990). Partial ordering of discrete life distributions based on ageing, Pak. J. Statistics, 6, 25-45. 
2. Adams G and Watson. R. (1989). A discrete time parametric model for the analysis of failure time data, Aust. J. Statistics 31, 365-384.

3. Bain. L.J. (1991). Statistical Analysis of Reliability and Life Testing Models. Marcel Dekker, New York.

4. Barlow R. E. and Proschan F. (1975) Statistical Theory of Reliability and Life Testing. Probability Models. Holt, Rinehart and Winston, New York.

5. Cai, J., Kalashnikov, V. (2000). NWU property of a class of random sums. Journal of Applied Probability 37, 283-289.

6. Cai, J., Willmot, G. (2005). Monotonicity and aging properties of random sums. Statistics and Probability Letters 73, 381-392.

7. Ebrahimi, N. (1986). Classes of discrete decreasing and increasing meanresiduallife distributions. IEEE Transactions on Reliability 35, 403-405.

8. Fagiuoli, E., Pellerey, F. (1994). Preservation of certain classes of life distribution under Poisson shock models. Journal of Applied Probability 31, 458-465.

9. Hu, T., Ma, M., Nanda, A.K. (2003). Moment inequalities for discrete aging families. Communication in Statistics, Part A Theory and Methods 32, 61-91.

10. Johnson, M.L., Kotz, S. and Kemp, A.W. (1993). Univariate Discrete Distributions, second ed. John Wiley \& Sons, INC, New York.

11. Karlin, S. (1968). Total Positivity. Vol. I. Standford University Press, Stanford C A.

12. Kijima, M. (2003). Stochastic Processes with Applications to Finance. Chapman \& Hall/ CRC, Boca Raton.

13. Nanda, A.K and Sengupta, D (2005). Discrete life distribution with decreasing reversed hazard, Sankhya-A, 55, 164-168.

14. Roy D and Gupta R. P. (1992). Classification of discrete lives, Microelectronic. Reliabil. 32, 1459-1473.

15. Salvia, A. A. (1996). Some results on discrete mean residual life. IEEE transactions on Reliability. 45, 359-361.

16. Salvia, A. A. and Bollinger, R. C. (1982). On discrete hazard functions. IEEE transactions on Reliability R-31(5), 458- 459.

17. Shaked, M., Shanthikumar, J.G. (1994). Stochastic Orders and their Applications. Academic Press, New York.

18. Shaked, M., Shanthikumar, J.G., Valdez-Torres, J.B. (1995). Discrete hazard rate functions. Computers and Operation Research 22, 391-402.

19. Willmot, G.E., Cai, J. (2001). Aging and other distributional properties of discrete compound geometric distributions. Insurance: Mathematics and Economics 28, 361-379.

20. Willmot, G.E., Drekic, S., Cai, J. (2005). Equilibrium compound distributions and stop-loss moments. Scandinavian Actuarial Journal 1, 6-24.

21. Willmot, G.E., Lin, X.S. (2000). Lundberg Approximations for Compound Distributions with Insurance Applications. Springer, New York.

22. Xekalaki, E. (1983). Hazard functions and life distributions in discrete time, Communs Statist. Theory Meth. 12, 2503-2509. 